\title{
Familiar size and the theory of off-sized perceptions
}

\author{
WALTER C. GOGEL \\ University of California, Santa Barbara, California \\ and \\ JOSÉ APARECIDO DA SILVA \\ University of São Paulo, Ribeirão Preto, Brazil
}

\begin{abstract}
The role of familiar size in the visual judgment of size and distance was investigated using reduced conditions of observation and instructions that called for either objective or apparent size and distance judgments. Each observer was presented with one object at one distance (56, 107 , or $149 \mathrm{~cm}$ ). This object, which was of constant physical size, was either a normal-sized playing card or a blank rectangle of the same size as the playing card. Distance responses were yerbal and size responses were tactual. For the blank rectangle, reported distance remained constant, whereas judged size decreased as physical distance increased for both objective and apparent instructions. For the playing card, reported distance increased as physical distance increased for both objective and apparent instructions. The judged size for the playing card, however, remained constant with objective instructions but decreased with apparent instructions as the physical distance increased. This pattern of results is consistent with the theory that, to a substantial degree, familiar size contributes to spatial responses by means of a cognitive process based upon familiar objects' appearing as smaller or larger than normal. Under conditions in which the familiar objects are normal in size (as in the present experiment), this process acts to correct a direct response to distance for any errors that might be present in the perception of distance.
\end{abstract}

\section{Familiar Size and the Size-Distance Invariance Hypothesis}

To investigate whether the characteristic size of a familiar object provides an effective cue to its distance from an observer, (1) verbal reports of distance often are used to measure perceived distance, and (2) cues to distance other than that of familiar size are reduced by having the observer view the familiar object monocularly in an otherwise dark visual field, with accommodation held constant. It has been found, under these conditions, that the observer's report of the distance of a familiar object often varies directly with its physical distance, if the familiar object is normal in size, or with its simulated distance, if the familiar object is larger or smaller than normal, where simulated distance is the distance at which the familiar object of normal size would need to be placed in order to subtend a particular visual angle (Epstein, 1965; Fitzpatrick, Pasnak, \& Tyer, 1982; Gogel \& Mertens, 1967; Ittelson, 1951). Results of this kind are inter-

The preparation of this paper was supported by the United States Public Health Service, Grant MH 39457, from the National Institute of Mental Health, to Walter C. Gogel and by Grants 82/0033-6 and 84/1632-6 from the Fundação de Amparo a Pesquisa do Estado de São Paulo (FAPESP), Brazil, to José Aparecido Da Silva.

W. C. Gogel's mailing address is: Department of Psychology, University of California, Santa Barbara, CA 93106. J. A. Da Silva's mailing address is: Department of Psychology, University of Sāo Paulo, Ribeirāo Preto, 14.100 Brazil. preted as being consistent with the notion that familiar size is an effective cue of distance and can be summarized by the size-distance invariance hypothesis (Epstein, Park, \& Casey, 1961; Gilinsky, 1951; Kilpatrick \& Ittelson, 1953; Schlosberg, 1950), which, in simple form, is:

$$
S^{\prime} / D^{\prime}=\tan \theta,
$$

where $S^{\prime}$ and $D^{\prime}$ are the perceived size and perceived distance of the familiar object, and $\theta$ is its visual angle. According to the size-distance invariance hypothesis (SDIH), familiar size will determine perceived distance $\left(D^{\prime}\right)$ for a given visual angle $(\theta)$ only if it determines perceived size $\left(S^{\prime}\right)$.

An explanation of the effect of the familiar size cue upon a direct response to distance, such as a verbal report of distance, is not necessarily limited to the perceptual factors $S^{\prime}$ and $D^{\prime}$ of Equation 1. An alternative paradigm, called the theory of off-sized perceptions, postulates that cognitive in addition to perceptual factors can contribute to direct judgments of size and distance (Gogel, 1969, 1974,1976 ). This theory, to be summarized below and applied in the present study, is considered more fully in a review by Gogel and Da Silva (1987).

\section{The Effectiveness of Cue Reduction}

In the present study, a single normal-sized familiar object (a playing card) was presented, to different groups 
of observers, at different distances in a visual alley. Except for the playing card, which was viewed monocularly through a restrictive aperture, the visual alley was completely dark. Under these conditions, extraneous cues to changes in the distance of the playing card from the observer, that is, relative distance cues other than the possible distance cue provided by familiar size, are likely to be completely eliminated. However, extraneous cues indicating that the playing card is at a constant distance from the observer are not completely eliminated. Instead, it has been found that, as cues to distance are increasingly removed, a target increasingly tends to appear at a distance from the observer of about 2 or $3 \mathrm{~m}$ regardless of its actual physical distance (Gogel \& Tietz, 1973). This has been called the specific distance tendency. In addition to this tendency, residual oculomotor cues of distance also seem to be unavoidably present. Although monocular observation and a small restrictive viewing aperture can eliminate the changes in accommodation (and accommodative convergence) that normally occur for objects at different physical distances, the accommodation will then be to a constant resting state of about $67 \mathrm{~cm}$ (Hennessey, Iida, Shiina, \& Leibowitz, 1976; Owens \& Leibowitz, 1976, 1983). Oculomotor resting states, together with the specific distance tendency, result in a composite distance factor called the egocentric reference distance (Gogel, 1972; Mershon \& Lembo, 1977). Thus, under the above conditions, in testing the effectiveness of the familiar size cue to distance by placing a familiar object of normal size at different distances from the observer, there are two conflicting sources of perceptual information regarding size and distance that must be considered. One is the constant value of the egocentric reference distance. The other is the possible effect of the familiar size cue to distance. Consider what the perception of the size and distance of the familiar object would be under these conditions if (1) the familiar size cue completely dominated the perception of distance, (2) the egocentric reference distance completely dominated the perception of distance, or (3) familiar size and the egocentric reference distance both contributed to the perception of distance. In the first alternative, the perceived distance of a normalsized familiar object always would be equal to its physical distance and the perceived size always would be equal to its familiar size. In the second alternative, the perceived distance of the familiar object always would be at the constant egocentric reference distance and its perceived size would be directly proportional to its retinal size, that is, inversely proportional to its physical distance. In the third alternative, the perceived distance of the familiar object would increase somewhat with physical distance, but less rapidly than the physical distance, and its perceived size would decrease somewhat with physical distance. What the theory of off-sized perceptions asserts is that direct reports of the size and distance of the familiar object often can resemble the results expected from the first alternative (familiar size completely effective), even though the perceptions actually are those of the second or third alternative (familiar size completely or partially ineffective).

\section{Application of the Theory of Off-Sized Perceptions}

There is no doubt that the familiar size of an object can affect a direct report of its distance. This is found even when the information as to the familiar size is communicated to the observer by suggestion from the experimenter rather than by its presence in the immediate stimulus (Gogel, 1981; Hastorf, 1950; Ittelson, 1951; Park \& Michaelson, 1974). The issue, however, is whether this effect on reported distance occurs because the familiar size (to be labeled $S_{\mathrm{c}}$ ) determines the perceived size $\left(S^{\prime}\right)$, which then determines a perceived distance $\left(D^{\prime}\right)$ in agreement with Equation 1. Assume, for the purpose of considering this issue, that the familiar size of the normalsized object located at different distances from the observer is completely unable to determine perceived size and perceived distance as described in the second alternative above. In that case, the $D^{\prime}$ of the familiar object would be at the egocentric reference distance which, together with $\theta$, would determine $S^{\prime}$ (Equation 1). It follows that the familiar object would be perceived as being smaller or larger in size than its familiar size as its physical distance was greater or less, respectively, than its constant perceived distance as produced by the egocentric reference distance. This perception or judgment of the object as having a perceived size $\left(S^{\prime}\right)$ that differs from its known or familiar size $\left(S_{\mathrm{c}}\right)$ is called an off-sized perception or an off-sized judgment. ${ }^{1}$ An off-sized perception or judgment $\left(S_{\mathrm{c}} / S^{\prime} \neq 1\right)$ provides the observer with information that the physical distance of the object is greater or less than the perceived distance of the object. In other words, the observer assumes that an object that appears to be smaller than normal $\left(S_{\mathrm{c}} / S^{\prime}>1\right)$ must be at a greater distance, and an object that appears to be larger than normal $\left(S_{\mathrm{c}} / S^{\prime}<1\right)$ must be at a shorter distance than the distance $\left(D^{\prime}\right)$ at which it is perceived to be. Or, in equation form,

$$
D_{\mathrm{c}}=D^{\prime}\left(S_{\mathrm{c}} / S^{\prime}\right) \text {. }
$$

The terms $S^{\prime}$ and $D^{\prime}$ in Equation 2 are perceptual, and their interrelation is specified by Equation 1. The term $S_{c}$ in Equation 2 is cognitive, since it requires the storage and retrieval of an internal representation of the object (a memory). In the case of a familiar object, $S_{\mathrm{c}}$ is the remembered size of the familiar object. The term $D_{\mathfrak{c}}$, from off-sized perceptions resulting from familiar size, usually is also cognitive (see Gogel \& Da Silva, 1987). Considering both Equation 1 and Equation 2, it is evident that the observer has two sources of information on which to base a direct response to size and distance. One, a primary source, specifies $S^{\prime}$ and $D^{\prime}$ consistent with Equation 1 . The other, a secondary source, specifies $S_{\mathrm{c}}$ and $D_{\mathrm{c}}$ consistent with Equation 2. Suppose that the response to size is completely determined by familiar size, $S_{\mathrm{c}}$. In this case, 
the constant familiar size of the object will be reported at all distances of the familiar object. Also, if the distance report is completely determined by $D_{\mathrm{c}}$, the reported distance always will equal the physical distance. The latter conclusion follows from substituting $S^{\prime}=D^{\prime} \tan \theta$ from Equation 1 in Equation 2, which results in

$$
S_{\mathrm{c}} / D_{\mathrm{c}}=\tan \theta
$$

But, since $\tan \theta=S / D$, if the physical size of the object, $S$, equals its familiar size, $S_{\mathrm{c}}$, then the cognitive distance, $D_{\mathrm{c}}$, will equal the physical distance, $D$. In other words, if the secondary factors $S_{\mathrm{c}}$ and $D_{\mathrm{c}}$ are present and completely dominate the observer's direct responses to size $\left(S_{\mathrm{R}}\right)$ and distance $\left(D_{\mathrm{R}}\right)$, both $S_{\mathrm{R}}$ and $D_{\mathrm{R}}$ will be veridical. If these responses are interpreted by the experimenter as representing perceived size and perceived distance, the experimenter will conclude (erroneously) that familiar size is an effective and veridical cue of both perceived size and perceived distance. Actually, however, for the situation described above, since perceived size and perceived distance were determined by the egocentric reference distance, familiar size was totally ineffective as a cue to either perceived size or perceived distance. The normal-size familiar object presented at different distances would, in this case, have been perceived to be at a constant distance (the egocentric reference distance) and to have had a perceived size proportional to its changing visual angle. Equation 3 has the same form as Equation 1, but, unlike Equation 1 , it involves cognitive, not perceptual, factors. It might be called the cognitive SDIH. If the effect of familiar size upon $S_{\mathrm{R}}$ and $D_{\mathrm{R}}$ occurs exclusively as a consequence of $S_{\mathrm{c}}$ and $D_{\mathrm{c}}$, as indicated by Equation 3, the familiar size cue cannot be regarded as a cue to perceived distance.

The difficulty in evaluating the familiar size cue to distance by obtaining direct responses of size and distance when differing primary and secondary sources are both available (i.e., whenever $S_{\mathrm{c}} \neq S^{\prime}$ ) can be expressed more generally. In such cases, it is likely that direct reports of size and distance often will be influenced by both sources. The direct reports of distance $\left(D_{\mathrm{R}}\right)$ will be determined by the relative weights $(a$ and $1-a$ ) given by the observer to $D^{\prime}$ and $D_{\mathrm{c}}$, respectively. The direct reports of size $\left(S_{\mathrm{R}}\right)$ will be determined by the relative weights $(b$ and $1-b)$ given by the observer to $S^{\prime}$ and $S_{\mathfrak{c}}$, respectively (Gogel, 1976). In equation form,

$$
D_{\mathrm{R}}=a D^{\prime}+(1-a) D_{\mathrm{c}}
$$

and

$$
S_{\mathrm{R}}=b S^{\prime}+(1-b) S_{\mathrm{C}} .
$$

It can be shown (see Gogel \& Da Silva, 1987) that if, and only if, $a=b$, will

$$
S_{\mathrm{R}} / D_{\mathrm{R}}=\tan \theta .
$$

Equation 6 might be called the response SDIH, since it involves a ratio of responses to size and distance. It is limited in its validity to the condition that $a=b$ in Equa- tions 4 and 5. If $a=b=0$, then $D_{\mathrm{R}}=D_{\mathrm{c}}$ and $S_{\mathrm{R}}=S_{\mathrm{c}}$, so that Equation 6 becomes identical to Equation 3. If $a=b=1$, then $D_{\mathrm{R}}=D^{\prime}$ and $S_{\mathrm{R}}=S^{\prime}$, so that Equation 6 becomes identical to Equation 1. Only in the latter case can $D_{\mathrm{R}}$ and $S_{\mathrm{R}}$ data provide a valid test of familiar size as a cue to perceived size and perceived distance.

\section{Differentiating Between the Effects of Primary and Secondary Information}

From the previous discussion it is clear that it is important, in testing the familiar size cue by obtaining such direct responses as verbal reports of size and distance or adjustments of comparison objects, to be able to evaluate the relative contributions of primary and secondary sources to the responses. There are several ways to accomplish this. One is to compare direct and indirect measures of perceived distance. Indirect measures, such as the head-motion procedure, for measuring perceived distance (see Gogel \& Da Silva, 1987), unlike direct measures, such as verbal reports, avoid the possibility that the observer will modify the distance response by using off-sized perceptions in the interest of being physically accurate. Another method, to be tried in the present study, is to compare the direct responses obtained from apparent instructions with those obtained from objective instructions. It is assumed that, compared to the effect of objective instructions, apparent instructions will increase the weight given to perceived as opposed to cognitive information, permitting, to some extent at least, the identification of the cognitive contribution.

\section{THE EXPERIMENT}

A study by Gogel (1976), which used the head-motion procedure as well as verbal reports of distance, concluded that, although familiar size is a cue of perceived distance, it is not a very effective cue. On the other hand, a more recent study by Fitzpatrick et al. (1982) asserts that familiar size is essentially a veridical cue of size and distance, at least for distances near the observer. The Fitzpatrick et al. study (1982) used both nonfamiliar (geometrical) and familiar objects, from which direct responses of size and distance were obtained. The nonfamiliar objects were octagonal white figures. The familiar objects were three-of-clubs playing cards. Either the same or different sizes of the octagons or of the playing cards were presented one at a time at the same or different distances to different groups of observers. The resulting judgments of size and distance from the octagons permitted the experimenters to determine that, in the absence of familiar size, no effective cues of distance were available in the visual alley to produce the changes in reported distances. Thus, if changes occurred in the reported distances of the playing cards as a function of their visual angles, these changes must be attributed to the cue of familiar size. The results provide clear evidence, seemingly in agreement with Equation 1, that a direct report of the distance of a familiar object is inversely related to its visual angle. 
According to Fitzpatrick et al., at distances not too far from the observer, the visual angle of a familiar object is an effective and veridical cue to both perceived size and perceived distance.

However, the study by Fitzpatrick et al. (1982) does not exclude an explanation in terms of an egocentric reference distance that produces off-sized perceptions of the familiar object, with the direct estimates of size and distance being the result mainly of $S_{\mathrm{c}}$ and $D_{\mathrm{c}}$ (Equation 3 ) rather than $S^{\prime}$ and $D^{\prime}$ (Equation 1). To clarify this problem of interpretation, again suppose that the perceived distances of all stimuli, whether octagonal figures or playing cards, had been completely determined by the egocentric reference distance and, therefore, were essentially constant. In this case, according to Equation 1 , since $D^{\prime}$ was constant, perceived size would have varied directly with the visual angle for both the octagons and playing cards. The stimulus objects of the same physical size at different distances would have appeared larger or smaller, depending upon whether they were physically closer or physically more distant, respectively, than the egocentric reference distance. Because the octagons had no familiar size, only $S^{\prime}$ and $D^{\prime}$ were available with these objects and thus only $S^{\prime}$ and $D^{\prime}$ were reported. The familiar size of the playing cards, on the other hand, provided a known size $\left(S_{c}\right)$ that differed from the $S^{\prime}$ produced by the egocentric reference distance, thus resulting in an off-sized judgment, which, in turn, resulted in a $D_{\mathrm{c}}$ that differed from $D^{\prime}$. However, Equation 6 would be satisfied if responses determined by $S^{\prime}$ and $D^{\prime}$ exclusively, by $S_{c}$ and $D_{c}$ exclusively, or by some combination of both were used, as long as $a=b$ in Equations 4 and 5. That is, $a=b=1$ if $S^{\prime}$ and $D^{\prime}$ were used exclusively, $a=b=0$ if $S_{\mathrm{c}}$ and $D_{\text {c }}$ were used exclusively, and $a=b=.5$ if both the primary and secondary sources of information contributed equally to $S_{\mathrm{R}}$ and $D_{\mathrm{R}}$. The crucial question for the Fitzpatrick et al. (1982) results is whether $S^{\prime}$ was determined by the egocentric reference distance or by the familiar size, that is, whether $S_{\mathrm{c}}=S^{\prime}$. The instructions to the observers in that study were to estimate the size (and distance) of the stimuli. In the case of the playing card, it seems likely that the observers interpreted this to mean that if perceived and familiar size differed, they were to respond with the familiar (remembered) size and not the perceived size. If this occurred, it is likely that it would be revealed in the different results obtained in the present experiment using the "apparent" and "objective" size instructions.

In the present study, unlike the Fitzpatrick et al. (1982) study, the physical size of the familiar or nonfamiliar stimulus presented at the different distances was always constant. This was either a normal-sized (seven-of-spades) playing card or a rectangle of the same physical size as a normal playing card. A second, more major difference was that the observer was instructed to respond to either apparent or objective characteristics, with the distinction between these indicated by examples. A third difference was that an alley containing a variety of cues to distance (a calibration alley) was used to calibrate the verbal reports of distances in feet or inches in an attempt to remove individual differences in the remembered size of a foot ruler (Gogel \& Tietz, 1973; Mershon, Kennedy, \& Falacara, 1977).

\section{Method}

\section{Observers}

The observers were 120 students ( 59 women and 61 men), whose participation in the experiment partially fulfilled a requirement of an introductory course in psychology. Their average age was 19 years. All had a visual acuity of $20 / 20$ in each eye, near and far, and a stereoscopic acuity of at least 25.25 " of arc, as measured in a Keystone Orthoscope. All were naive regarding the purposes of the experiment.

\section{Apparatus}

The room in which the experiment was conducted contained two visual alleys, in each of which the illumination was independently controlled. One alley, which was totally dark during the observations except for the presentation of the single stimulus, was used only for the experimental situations. The other, which was lighted by a series of overhead lights, was used only for the calibration situation. Each alley had its own observation position consisting of a chinrest and a large viewing aperture with an occluding shutter. The observation positions were in a lightproof booth that could be completely darkened. A speaker and microphone allowed the experimenter and observer to communicate at each observation position.

Experimental situations. In the experimental situation, either the familiar object or the blank white rectangle was presented at one of three distances from the observer, under conditions in which only the single object was perceived in an otherwise totally dark alley. The alley was $61 \mathrm{~cm}$ wide and $800 \mathrm{~cm}$ long, and was lined with black velveteen to eliminate stray light. The familiar object was a positive transparency of a normal-sized seven-of-spades playing card $(5.7 \mathrm{~cm}$ wide and $8.9 \mathrm{~cm}$ high) oriented with its largest dimension vertical. The blank white rectangle also was a positive transparency with the same dimensions as the playing card. The playing card and blank rectangle were transilluminated by white light from a diffuse electroflourescent source-a light box with a restricted luminous surface located immediately behind the stimulus object. A light-tight holder attached to the front of the box permitted the experimenter to insert either the playing card or the blank rectangle transparency. The playing card or blank rectangle, always of constant physical size, was presented perpendicular to the median plane of the observer's right eye and was centered at the height of the observer's right eye at a distance from the observer of 56,107 , or $149 \mathrm{~cm}$. The average luminance of the white rectangle and the white portions of the playing card was $2.5 \mathrm{fL}$. Each observer was presented with only one of the stimulus objects (the playing card or blank rectangle) at only one of the three distances. The object was viewed monocularly, through a $0.6-\mathrm{mm}$ pinhole in a mask worn by the observer with the left eye occluded. A shutter could be lowered to occlude a large $(36 \times 15 \mathrm{~cm})$ viewing aperture at the observation position. When the light in the observation booth was on, a white mark on the shutter assisted the observer in aligning the pinhole so that the entire stimulus object could be seen through the pinhole when the light in the observation booth was turned off and the shutter was raised. Considerable care was taken so that when the shutter was raised nothing except the single stimulus object was visible to the observer anywhere.

The observer had two tasks. One was to indicate verbally the apparent or objective distance of the object. The other was to indi- 
cate its apparent or objective size. The observer accomplished the latter task by adjusting the lateral separation between the inner surfaces of two square rods to duplicate the judged width of the object. The left rod was stationary, and the right rod was movable. The rods were located directly below the head of the observer, but a small curtain kept them from being seen whether or not the observation booth was lighted. The rod adjustment was made in the dark, completely by touch.

Calibration situation. The calibration of the observer's verbal reports of distance was accomplished in a visual alley, $95 \mathrm{~cm}$ wide and $290 \mathrm{~cm}$ long, with a checkerboard pattern of red and white squares $(2.5 \mathrm{~cm}$ on a side) covering the alley floor. The floor of the alley was $20.5 \mathrm{~cm}$ below the height of the chinrest positioned at the front of the alley. The walls of the alley were formed by white cloth. Six white cardboard squares, $10 \mathrm{~cm}$ on a side, stood upright on the alley floor, at $40,70,120,170,230$, and $270 \mathrm{~cm}$ from the observer, in such a way that no square occluded any part of another when viewed from the observation position. A number from 2 to 7 (randomly selected) was painted in black on each square so that the experimenter could identify each square to the observer. The viewing in the calibration alley was always binocular through an aperture ( $56 \mathrm{~cm}$ wide $\times 40 \mathrm{~cm}$ high) that could be occluded by a shutter. The entire calibration alley was visible throughout its length during the calibration procedure.

\section{Procedure}

Experimental situations. The observers were assigned randomly to 12 groups of 10 observers each. Each group was presented with either the playing card or the blank rectangle at only one distance with instructions to respond to either apparent size and apparent distance or objective size and objective distance. For half the observers, the response to size $\left(S_{R}\right)$ was obtained before the response to distance $\left(D_{\mathrm{R}}\right)$. For the remaining observers, this order was reversed. To delineate the distinction between the apparent and objective responses, the observers were given the following illustrations:

\begin{abstract}
To illustrate the possible difference between apparent and physical distance recall that, on a clear day, mountains often appear close, whereas you might know or guess that physically they are far away. On the other hand, it is possible that an object might appear far away even though you know or guess that it is close. On an overcast day, the mountains might appear farther away than you might know or guess them to be....

To illustrate the possible difference between apparent and physical width consider the situation in which you are in an airplane high in the air. When viewing a house from the airplane, it might appear to be as small as a toy house even though you know that physically it is large. On the other hand, an object might appear to be large, even though you know or guess that it is smaller than it appears. For example, if you are looking through a microscope in a biology class, the object at which you are looking might appear as large as from $1 / 2$ to 1 inch even though you know it is physically so small as to be visible only through a microscope.
\end{abstract}

The observers who were to respond to physical characteristics were informed that they were to indicate the physical distance and physical width of the object, not the apparent distance and apparent width of the object, if the physical and apparent differed. The observers who were to respond to apparent characteristics were told that they were to indicate the apparent distance and apparent width of the object, not the physical distance and physical width, if the physical and apparent differed.

The response to distance consisted of a single verbal report of distance in feet or inches or in some combination of both. The response to width was the average of two successive adjustments of the separation of the posts by touch.
After completing the response to size and distance, each observer completed the following two additional tasks at the experimental observation position in the following order. For the first of these, the shutter on the large viewing aperture was closed. (1) Observers who had been presented with the playing card used the rods to duplicate, by touch, their remembered width of a normal playing card. Under the same conditions, observers who had been presented with the blank rectangle used the rods to indicate their remembered width of $21 / 4$ in. Each of these adjustments was completed twice with the booth lights off and with no stimulus visible. (2) For the second of these tasks, the shutter was closed, the booth light was turned on, and the experimenter entered the booth bringing with him a playing card or a blank white rectangle identical in size to the stimulus object presented previously. Then the mask containing the pinhole was removed, and the observer was shown the card or rectangle (standard) and told to assign it a value of 10 . The mask was then replaced, the booth lights were turned off, the shutter was raised, and the observer, again viewing the stimulus object (playing card or blank rectangle) at the distance at which it had been presented previously, assigned to it a number reflecting its apparent size relative to that of the standard. The instructions for this task asked for apparent size regardless of whether the previous instructions to that observer had been for apparent or objective judgments.

Calibration situation. After completing these tasks, the observer removed the mask and moved to the observation position in front of the calibration alley. The lights in the calibration alley were turned on and the shutter was opened to reveal the six numbered squares presented simultaneously on the alley floor. The observer was instructed to report verbally in feet or inches, or some combination of both, the distance to each of the numbered squares in a prearranged random order that was different for each observer. Observers who had been instructed for the first tasks in the experimental situation to make apparent (or objective) distance judgments also were told to make apparent (or objective) distance judgments in the calibration situation.

\section{Results}

The results from the 12 groups in the experimental situations are given in Table 1 . The distance responses are labeled $D_{R}$, the size responses $S_{R}$, and the transformation of $D_{R}$ by the data from the calibration alley is labeled $D^{\prime}{ }_{\mathrm{R}}$. The method for converting $D_{\mathrm{R}}$ scores to $D^{\prime}{ }_{\mathrm{R}}$ scores was as follows. A power function relating the reported to the physical distances of the squares in the calibration alley was computed by converting these values to logarithms and obtaining a linear line of best fit using the method of least squares. Since many cues of distance were available in the calibration alley, it was assumed that physical distance was correctly perceived, thus allowing the physical distance of the numbered cards to represent perceived distance. Assuming that this substitution of perceived distance for physical distance is valid, the equation found by this procedure expresses the relationship between perceived and reported distance. This equation can then be applied to the distances reported in the experimental situations, converting these reported distances to perceived distances (Mershon, Kennedy, \& Falacara, 1977). It is this transformation, calculated from the observer's data in the calibration alley and applied to the same observer's data in the experimental situations, 
Table 1

Results From Experimental Situations

\begin{tabular}{|c|c|c|c|c|c|}
\hline \multirow[b]{2}{*}{ Instructions } & \multirow[b]{2}{*}{ Responses } & \multirow{2}{*}{$\begin{array}{l}\text { Physical } \\
\text { Distance }\end{array}$} & \multicolumn{3}{|c|}{ Results in Centimeters } \\
\hline & & & Mean & Median & $S D$ \\
\hline \multicolumn{6}{|c|}{ Playing Card } \\
\hline \multirow[t]{3}{*}{ Apparent } & $S_{\mathbf{R}}$ & $\begin{array}{r}56 \\
107 \\
149\end{array}$ & $\begin{array}{l}3.03 \\
2.44 \\
1.89\end{array}$ & $\begin{array}{l}3.00 \\
2.50 \\
1.70\end{array}$ & $\begin{array}{l}0.50 \\
1.20 \\
0.80\end{array}$ \\
\hline & $D_{\mathrm{R}_{\mathrm{f}}}$ & $\begin{array}{r}56 \\
107 \\
149\end{array}$ & $\begin{array}{r}48.3 \\
125.2 \\
162.6\end{array}$ & $\begin{array}{r}45.7 \\
91.5 \\
122.0\end{array}$ & $\begin{array}{r}25.0 \\
119.4 \\
170.0\end{array}$ \\
\hline & $D_{\mathrm{R}}^{\prime}$ & $\begin{array}{r}56 \\
107 \\
149\end{array}$ & $\begin{array}{r}54.2 \\
168.9 \\
160.2\end{array}$ & $\begin{array}{c}48.9 \\
102.5 \\
132.1\end{array}$ & $\begin{array}{r}21.2 \\
182.2 \\
137.8\end{array}$ \\
\hline \multirow[t]{4}{*}{ Objective } & $S_{\mathbf{R}}$ & $\begin{array}{r}56 \\
107 \\
149\end{array}$ & $\begin{array}{l}4.94 \\
5.25 \\
4.27\end{array}$ & $\begin{array}{l}4.90 \\
5.40 \\
4.40\end{array}$ & $\begin{array}{l}1.10 \\
1.50 \\
0.90\end{array}$ \\
\hline & $D_{\mathrm{R}}$ & $\begin{array}{r}56 \\
107 \\
149\end{array}$ & $\begin{array}{r}70.1 \\
86.9 \\
134.2\end{array}$ & $\begin{array}{r}45.7 \\
76.2 \\
122.0\end{array}$ & $\begin{array}{l}55.3 \\
52.8 \\
94.7\end{array}$ \\
\hline & $D_{\mathrm{R}}^{\prime}$ & $\begin{array}{r}56 \\
107 \\
149\end{array}$ & $\begin{array}{r}81.7 \\
104.3 \\
158.9\end{array}$ & $\begin{array}{r}55.6 \\
100.5 \\
142.6\end{array}$ & $\begin{array}{r}72.7 \\
40.9 \\
107.3\end{array}$ \\
\hline & \multicolumn{5}{|c|}{ Blank Rectangle } \\
\hline Apparent & $S_{\mathrm{R}}$ & $\begin{array}{r}56 \\
107 \\
149\end{array}$ & $\begin{array}{l}2.90 \\
2.37 \\
1.71\end{array}$ & $\begin{array}{l}2.63 \\
1.60 \\
1.53\end{array}$ & $\begin{array}{l}1.40 \\
1.60 \\
0.50\end{array}$ \\
\hline & $D_{\mathrm{R}}$ & $\begin{array}{r}56 \\
107 \\
149\end{array}$ & $\begin{array}{r}95.5 \\
80.0 \\
120.9\end{array}$ & $\begin{array}{l}30.5 \\
30.5 \\
33.0\end{array}$ & $\begin{array}{l}135.1 \\
109.8 \\
192.8\end{array}$ \\
\hline & $D_{\mathbf{R}}^{\prime}$ & $\begin{array}{r}56 \\
107 \\
149\end{array}$ & $\begin{array}{r}100.3 \\
98.7 \\
155.1\end{array}$ & $\begin{array}{l}38.3 \\
44.8 \\
39.7\end{array}$ & $\begin{array}{l}128.7 \\
140.8 \\
266.8\end{array}$ \\
\hline \multirow[t]{3}{*}{ Objective } & $S_{\mathrm{R}}$ & $\begin{array}{r}56 \\
107 \\
149\end{array}$ & $\begin{array}{l}2.46 \\
1.89 \\
1.94\end{array}$ & $\begin{array}{l}2.30 \\
1.83 \\
1.70\end{array}$ & $\begin{array}{l}1.20 \\
0.70 \\
1.00\end{array}$ \\
\hline & $D_{\mathrm{R}}$ & $\begin{array}{r}56 \\
107 \\
149\end{array}$ & $\begin{array}{l}49.5 \\
52.7 \\
58.1\end{array}$ & $\begin{array}{l}25.4 \\
45.7 \\
45.7\end{array}$ & $\begin{array}{l}61.6 \\
39.6 \\
47.3\end{array}$ \\
\hline & $D^{\prime} \mathbf{R}$ & $\begin{array}{r}56 \\
107 \\
149\end{array}$ & $\begin{array}{l}63.2 \\
62.7 \\
64.9\end{array}$ & $\begin{array}{l}30.5 \\
61.2 \\
53.9\end{array}$ & $\begin{array}{l}79.1 \\
44.8 \\
47.3 \\
\end{array}$ \\
\hline
\end{tabular}

Note-The size responses are labeled $S_{R}$, the distance responses are labeled $D_{\mathrm{R}}$, and the transformation of distance responses by the data obtained from the calibration situation are labeled $D_{\mathrm{R}}^{\prime}$.

which, when averaged over the 10 observers of a group, gives the $D^{\prime}{ }_{R}$ scores shown in Table 1 .

It is of some interest to consider whether the reports of distance in the calibration alley differed as a function of whether apparent or objective instructions regarding distance were used. The average relationship obtained was that $D_{\mathrm{R}}=.94 D^{1.00}$ for apparent instructions and $D_{\mathrm{R}}=$ $.85 D^{1.02}$ for objective instructions, with $D_{\mathrm{R}}$ the verbal report of distance and $D$ the physical distance of the numbered squares. According to the $t$ test, neither the .94-.85 difference nor the 1.00-1.02 difference was significant at the .05 level $[t \mathrm{~s}(118)=1.06$ and 1.25 , respectively].

The analysis of variance of the experimental data that follows is substantially the same whether the $D_{\mathrm{R}}$ or the $D^{\prime}{ }_{R}$ data of Table 1 is used; therefore, only the analysis of the $S_{\mathrm{R}}$ and $D_{\mathrm{R}}$ data, not the $D^{\prime}{ }_{\mathrm{R}}$ data, will be presented.
Since the results obtained from two observers in the experimental situation using the blank rectangle at a distance of $149 \mathrm{~cm}$, with objective instructions, were quite different from the results obtained from the remaining observers of that group, the data from these two observers were replaced by data obtained from two additional observers. If the replacement had not occurred, the results from the original group would have been $3.25,1.70$, and $4.70 \mathrm{~cm}$ for the mean, median, and $S D$ for $S_{\mathrm{R}}$ and 130.3, 40.6, and $276.6 \mathrm{~cm}$ for the mean, median, and $S D$ for $D_{R}$, respectively.

Both means and medians are presented in Table 1 because these are sometimes quite different. This is particularly the case for the $D_{\mathrm{R}}$ data obtained with apparent instructions and the blank rectangle. Because of this skewness of some of the data, a logarithmic transformation was used before the analysis of variance (ANOVA) was applied to any of the $D_{\mathrm{R}}$ or $S_{\mathrm{R}}$ results.

\section{Responses to the Blank Rectangle}

The reported distance of the blank rectangle under either apparent or objective instructions did not change significantly as a function of physical distance. This is shown by a two-way ANOVA in which neither instruction nor physical distance, or their interaction, had a significant effect upon the distance response $\left(D_{\mathrm{R}}\right)$ of the blank rectangle, at the .05 level $[F(1,54)=1.14, F(2,54)=.31$, and $F(2.54)=.16$, respectively]. From this it follows that the experimental situations in the present study were maximally reduced, and thus the study provides conditions that are appropriate for a test of the effectiveness of the familiar-size cue. The mean and particularly the median values of $D_{\mathrm{R}}$ for the blank rectangle, with either type of instructions, indicates that the egocentric reference distance was quite close to the observers.

According to Table 1, the size responses to the blank rectangle tended to decrease with increasing distance from the observer, but did not change appreciably as a function of instructions. A two-way ANOVA provides support for these conclusions. The effect of physical distance on the size response was significant $[F(2,54)=3.11, p=$ $.051]$, whereas the effect of instructions clearly was not significant $[F(1,54)=.74]$. Combining the instructions, a Kruskal-Wallis one-way ANOVA confirmed that the size response to the blank rectangle indeed was modified significantly by the physical distance $[H(2)=12.59, p<$ $.01]$. The reported size of the blank rectangle, averaged over the two types of instructions, was $2.21 \mathrm{~cm}$, which is not much smaller than the $2.45-\mathrm{cm}$ average size response for the playing card under the apparent instructions. An explanation for the lack of an effect of instructions upon the size responses to the blank rectangle is as follows. Because familiar size, $S_{\mathrm{c}}$, was not present, only perceived size, $S^{\prime}$, resulting from the visual angle and the egocentric reference distance, was available to the observers. Thus, the only option for the observers was to respond with $S^{\prime}$, with this response decreasing with distance (or visual angle) consistent with Equation 1. 
In summary, increases in the physical distance of the blank rectangle of constant physical size resulted in decreasing size responses, whereas the distance responses remained unchanged. This is consistent with the idea that the presence of an egocentric reference distance determined a constant perceived distance and, in turn, that this constant perceived distance, together with the decreasing retinal size, determined a decreasing perceived size consistent with the SDIH (Equation 1).

\section{Responses to the Playing Card}

Instructions modified the response to the width $\left(S_{R}\right)$ of the playing card, as shown in Table 1 . The significance of this result was tested by a two-way ANOVA of the $S_{R}$ data of the playing card. Instructions and physical distance were significant $[F(1,54)=62.9, p<.001$, and $F(2,54)=4.76, p=.012]$, but the interaction of instructions and physical distance was not significant $[F(2,54)$ $=2.02, p=.140]$. From a one-way ANOVA, changes in the response to the size of the playing card as a function of physical distance were significant with apparent instructions $[F(2,27)=3.99, p=.029]$ but not with objective instructions $[F(2,27)=1.51, p=.237]$. Objective instructions with the playing card resulted in size responses (an overall average of $4.87 \mathrm{~cm}$ ) that were similar to the remembered size of a playing card (an overall average of $4.82 \mathrm{~cm}$, as obtained subsequently) and were independent of the physical distance of the playing card from the observer. Apparent instructions, on the other hand, resulted in size responses to the playing card that were much smaller (an overall average of $2.45 \mathrm{~cm}$ ) and that decreased as the physical distance of the card increased.

An examination of the distance responses $\left(D_{R}\right)$ to the playing card shown in Table 1 reveals an increase in $D_{R}$ for each increase in physical distance for both apparent and objective instructions. This effect of physical distance is supported by a two-way ANOVA of the distance responses, in which it is found that physical distance was a significant factor $[F(2,54)=3.47, p=.037]$, but neither the factor of instructions nor the interaction of instructions and physical distance were significant $[F(1,54)$ $=.40, F(2,54)=.05$, respectively]. Unlike the effect of instructions on the response to the size of the playing card, the different instructions had no significant effect upon the response to the distance of the playing card.

In summary, the responses to the playing card under objective instructions are consistent with the idea that familiar size is an important factor in the response to size and distance. If it could be assumed that the responses were entirely perceptual, it could be concluded, in agreement with Fitzpatrick et al. (1982), that familiar size, together with visual angle, is an essentially veridical determiner of perceived size and perceived distance. Unfortunately for this interpretation, the size responses obtained under apparent instructions show that nonperceptual factors contributed to the responses to the playing card. Under apparent instructions, size responses to the play- ing card and the blank rectangle were essentially identical, whereas distance responses to the playing card were essentially the same for both types of instructions. The familiar size of the playing card failed to determine perceived size, and, therefore, it must also have failed to determine perceived distance. But, if familiar size was ineffective in determining perceived size, and hence necessarily ineffective in determining perceived distance, how is the clear increase in reported distance with an increase in physical distance that was obtained for both kinds of instructions to be explained? The explanation is that the smaller than normal perceived size of the playing card that resulted from perceiving the playing card of known size at a distance close to that specified by the egocentric reference distance produced an off-sized perception $\left(S_{\mathrm{c}} / S^{\prime}\right)$, which, for both the objective and apparent instructions, resulted in a cognitive response $\left(D_{\mathrm{c}}\right)$ to distance. In the case of the blank rectangle, however, familiar aspects of the stimulus were absent, and, since an offsized perception, and therefore $D_{\mathrm{c}}$, was not available, the response to the distance of the blank rectangle was perceptual and was determined by the egocentric reference distance. On the other hand, the apparent instructions seemed to have no effect upon the response to the distance of the familiar object and, thus, no effect upon whether $D_{\mathfrak{c}}$, rather than $D^{\prime}$, was used in the distance response.

The results from the two additional tasks following the presentation of a stimulus object to an observer in the main portion of the experimental situation were as follows: (1) The duplication of the width of a playing card $(5.7 \mathrm{~cm})$ from memory resulted in a mean, median, and standard deviation of $4.82,4.68$, and $1.01 \mathrm{~cm}$, respectively; the duplication from memory of 2.25 in. (also $5.7 \mathrm{~cm}$ ) resulted in values of $4.12,3.85$, and $1.42 \mathrm{~cm}$, respectively. (2) The magnitude estimations of the apparent size of the playing card or blank rectangle viewed under reduced conditions following the presentation of the standard (playing card or blank rectangle) presented under lighted conditions are shown in Table 2. An analysis of variance of a log transformation of the data of Table 2 indicated that the only significant factor was the physical distance of the stimulus object $[F(2,114)=6.26, p=.003]$. It is also clear from the magnitude estimations shown in Table 2 (averages of 6.01 for the playing card and 5.82 for the blank rectangle) that both the playing card and the blank rectangle were usually perceived to be considerably smaller than their respective standards $[t \mathrm{~s}(59)=7.13$ and 4.17 , respectively, $p<.001]$ with these standards presented under full-cue conditions and assigned a value of 10 . The results from the playing card provide evidence that the playing card was seen in the experimental situations to be a small off-sized object at each of the distances of presentation, with the perceived size of the playing card generally decreasing as the physical distance of the card increased. These results are in opposition to the view that familiar size is a veridical cue of perceived size and hence of perceived distance. 
Table 2

Magnitude Estimations of the Size of the Playing Card and Blank Rectangle Presented Under Reduced-Cue Conditions With the Standard (Modulus) Provided by the Playing Card and Blank Rectangle Presented Under Full-Cue Conditions and Assigned a Value of $\mathbf{1 0}$

\begin{tabular}{lccccccc} 
& \multicolumn{3}{c}{ Playing Card } & & \multicolumn{3}{c}{ Blank Rectangle } \\
\cline { 2 - 4 } \cline { 6 - 8 } Physical Distance: & $56 \mathrm{~cm}$ & $107 \mathrm{~cm}$ & $149 \mathrm{~cm}$ & & $56 \mathrm{~cm}$ & $107 \mathrm{~cm}$ & $149 \mathrm{~cm}$ \\
\hline Mean & 7.32 & 4.92 & 5.80 & & 9.25 & 4.27 & 3.95 \\
Median & 7.25 & 5.00 & 5.00 & & 5.00 & 3.50 & 4.50 \\
SD & 3.29 & 2.80 & 6.04 & & 12.54 & 2.62 & 2.26 \\
\hline
\end{tabular}

\section{DISCUSSION}

As described in Fitzpatrick et al.'s (1982) article, the instructions they used were similar to objective instructions. Therefore, the agreement between their results and the results obtained in the present study for the situation using the playing card and objective instructions are to be expected. These results apply to the cognitive SDIH of Equation 3, which is identical in form to the perceptual SDIH of Equation 1. The results from each of the four experimental situations are shown in Figure 1, in which $S_{\mathbf{R}} / D_{\mathbf{R}}$, computed from the medians of Table 1 , is plotted against the tangent of the visual angle, $\theta$, of the stimulus. Medians were used because in the case of the blank rectangle the distributions of 10 scores were often skewed. For either the perceptual SDIH (Equation 1) or the cognitive SDIH (Equation 3), the results in Figure 1 should fall on or near the dashed lines. In general, this happens for the cases of Figures 1A, 1C, and 1D, but it does not do so in the case of Figure 1B. Except for Figure 1B, Equation 6 provides an acceptable fit to the data. The question is whether the appropriate equation for Figure $1 \mathrm{~A}$ is Equation 1 or Equation 3.

It will be recalled that using apparent, rather than objective, instructions with the playing card resulted in a change in $S_{\mathrm{R}}$ from that expected from familiar size to a reported size similar to that obtained from the blank rectangle. It follows in the situation of Figure 1A, in which objective instructions were used, that, since familiar size did not determine perceived size, it also did not determine perceived distance, and $S_{\mathrm{R}}$ and $D_{\mathrm{R}}$ were determined mainly by $S_{\mathrm{c}}$ and $D_{\mathrm{c}}$, not by $S^{\prime}$ and $D^{\prime}$. Equation 3 , not Equation 1, is appropriate for Figure $1 \mathrm{~A}$. On the other hand, in the situation of Figure 1B, in which apparent instructions were used, $S_{\mathrm{R}}$ was determined mainly by $S^{\prime}$ (which varied directly with $\theta$ ) rather than by $S_{\mathrm{c}}$. However, in this case, $D_{\mathrm{R}}$ was not modified by the change to apparent instructions, and it continued to be determined by $D_{\mathrm{c}}$. This resulted in the lower $S_{\mathrm{R}} / D_{\mathrm{R}}$ values of Figure 1B, and consequently, the data fall considerably below the dashed line. Figure 1B is not appropriate to Equation 1, Equation 3, or Equation 6. It is, however, consistent with the condition that $a \neq b$. Presumably, in the case of Figure 1B, $a=0$ and $b=1$ in Equations 4 and 5. That is, $S_{\mathrm{R}}$ was determined by $S^{\prime}$ from the egocentric reference distance, whereas $D_{\mathrm{R}}$ was determined by $D_{\mathrm{c}}$ from the off-sized perceptions. On the other hand, the data of
Figures 1C and 1D apply to Equations 1 and 6 with $a=b=1$, since, in these cases, only $S^{\prime}$ and $D^{\prime}$ (not $S_{\mathrm{c}}$ and $D_{\mathrm{c}}$ ) were available to specify $S_{\mathrm{R}}$ and $D_{\mathrm{R}}$. The difference between Figure 1B and the remaining drawings of Figure 1 illustrates the need to determine whether a test of the familiar-size cue predominantly involves perceptual or cognitive factors or some combination of the two. The previous study (Gogel, 1976), which used the headmotion procedure and verbal reports, indicated that familiar size had some effect on perceived distance, but a large portion of the verbal response to distance was considered to be cognitive. The present study provides additional support for the latter portion of this conclusion. It cannot be concluded from the present study that the familiar-size cue was completely ineffective in determining perceived size and perceived distance. It can be concluded, however, from the effect of apparent instructions on the direct response to the size of the familiar object (as shown
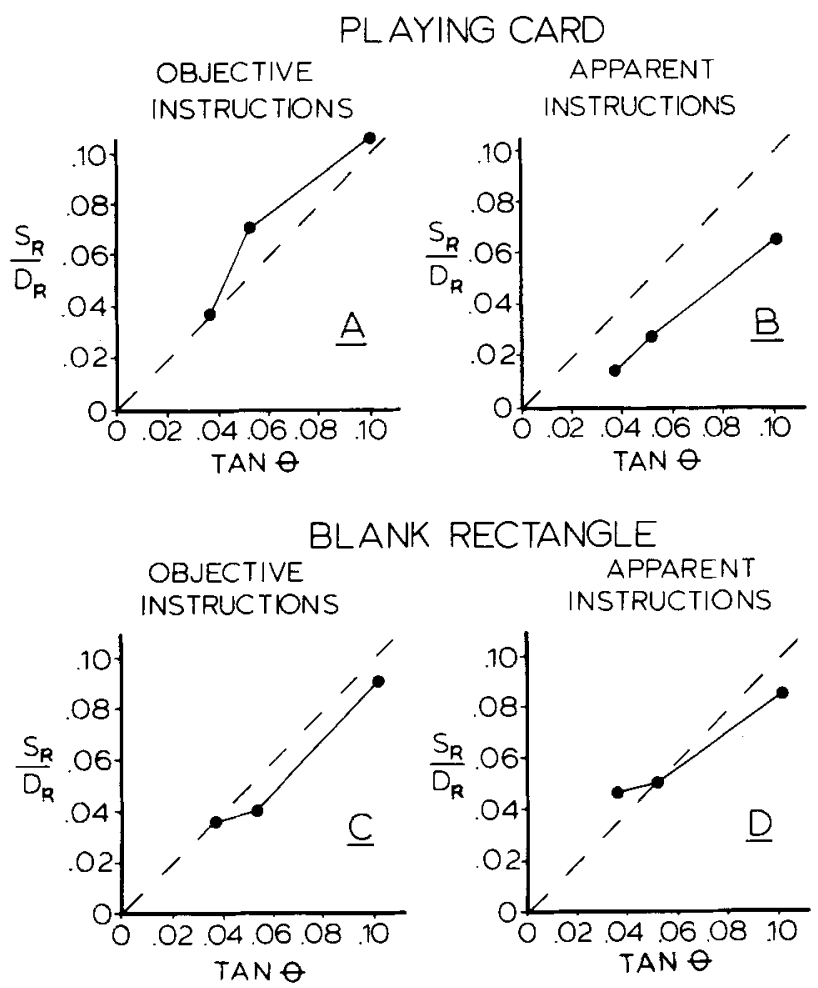

Figure 1. The relationship between $S_{\mathrm{R}} / D_{\mathrm{R}}$ and $\tan \theta$ using apparent or objective instructions and a familiar object (a playing card) or a nonfamiliar object (a blank rectangle). 
in Tables 1 and 2) that secondary (cognitive) processes as well as primary (perceptual) processes were involved in the responses obtained under objective instructions.

A recent article by Higashiyama (1984) examines the effect of familiar size upon verbal reports of size and distance as a function of viewing attitude and the level of opposing cues. Three images of the same physical width but differing in familiar size were presented at a constant physical distance to produce simulated distances of 43 , 99 , and $182 \mathrm{~cm}$. In one experiment, the instructions were to disregard the information from familiar size (apparent instructions) or to use that information (assumptive instructions) in the reports. In a second experiment, viewing attitude was classified, using interviews following the reports, as apparent or assumptive. From an examination of the data, it can be concluded that the apparent, as opposed to the assumptive, attitude resulted in a reduction in the range of size estimates obtained in both experiments under all cue conditions (monocular, binocular, or fullcue) and sometimes also a reduction in the range of distance estimates. Since the visual angle of the width of the familiar objects and their physical distances were constant, the present authors interpret these range reductions as being consistent with perceived size and perceived distance as being largely determined by the extraneous (opposing) cues and not by familiar size. The greater ranges of $D_{R}$ and particularly $S_{\mathrm{R}}$ under the assumptive attitude as compared with the apparent attitude can be attributed to offsized perceptions' providing some cognitive modification of $D_{R}$ by $D_{c}$ and of $S_{R}$ by $S_{c}$, under the assumptive attitude, consistent with the present study.

In two rather recent studies (Granrud, Haake, \& Yonas, 1985; Yonas, Pettersen, \& Granrud, 1982), it has been concluded that 7 -month-old infants can use familiar size as a cue to relative distance. In the first study, photographs of faces were the familiar objects. In the second study, object familiarity was established during $10 \mathrm{~min}$ of play with a pair of objects of different sizes. The finding that the ability to judge distance by using familiar size occurs in the 7-month-old infant seems to suggest that the familiar-size cue is the result of perceptual, not cognitive, processes. The question, however, is whether the ability to use this cue requires greater learning if cognitive, rather than perceptual, processes are involved. For the response to be perceptual, two kinds of association would need to be formed: (1) A perceived size would need to become associated with the particular stimulus configuration. (2) To use this learned size to determine which of two objects was closer in distance, a different perceived distance would need to become associated with each visual angle of the familiar object. The complexity of the latter learning would be reduced (considering the variety of familiar sizes available) if the associations were formed directly between perceived size per unit of retinal size as one factor and perceived distance as the other (see Gogel, 1964). Learning to use familiar size as a cognitive cue to indicate which of two objects is closer in distance may require no more and perhaps less learning. Again, a $S_{\mathrm{c}}$ would need to become associated with the particular stimulus configuration. The terms $S^{\prime}$ and $D^{\prime}$ in Equation 2 are the result of sensory information provided by extraneous or residual cues (perhaps the egocentric reference distance) and require no new associations. Thus, to have $D_{\mathrm{c}}$ available, the observer needs only to learn the rule that any object that appears larger or smaller than its normal size is at a distance less or greater, respectively, than its perceived distance. In view of the inability of adult observers to always consciously distinguish between $D_{\mathrm{c}}$ and $D^{\prime}$, as happened in the present study, it seems that $D_{\mathrm{c}}$ can be processed at a level below that of conscious awareness. The importance of $D_{\mathrm{c}}$ in achieving veridical judgments of distance from a knowledge of familiar size would be enhanced if it did not demand conscious judgments and could apply to animal as well as human responses.

Figures 1C and 1D show that under cue reduction the ratio of the size to the distance response for the blank rectangle for either kind of instruction is closely related to the visual angle. But it would be incorrect to interpret this as a direct response to the retinal image. The evidence for the egocentric reference distance or the specific distance tendency clearly is opposed to such an interpretation. Despite the cue reduction and an unfamiliar object, a distance response (in this case specified by the egocentric reference distance) always was present. This distance response, together with the retinal size, determined the response to size. Therefore, it is incorrect to state that under reduced conditions the size "estimates correspond to the retinal angle rather than the metric size of the stimuli" (Rix, Tyer, \& Pasnak, 1983, p. 29). Instead, the size estimates, in agreement with the SDIH (Equation 1), corresponded to the visual angle because they were responses to metric size, as determined by responses to metric distance, with the latter determined by the egocentric reference distance.

Rock (1977) and Rock and McDermott (1964) use the term "phenomenal extensity" to describe a proximal mode of response to size (also see Mack, 1978) that is similar to a direct response to visual angle and that, by implication, is not metric and does not involve a perception of distance. The question is whether a shift to an extensity response can explain the effect of apparent instructions upon the perceived size of the playing card in the present experiment. If so, an explanation in terms of a perceptual-cognitive size distinction would be unnecessary. There are reasons for rejecting an explanation based upon a change of mode. If responses in the proximal or extensity mode are assumed to occur in the present study, they must occur even though the size judgment requires a linear response - in this case, an adjustment of the lateral separation of the two measuring rods. Also, since the rods physically were at the position of the observer, and only the right rod was movable, it was not possible for the observer to make an angular judgment by aligning each rod beneath the visual line of sight to the right and left edges of the stimulus. But, even if both rods had been movable, it would be very unlikely that adjustments of the magni- 
tude obtained could be interpreted as angular responses, since the rod separation required at the observer's position for an angular response always would have been close to zero. In addition, the size responses to the blank rectangle were not different for apparent and objective instructions, suggesting that the difference in instruction did not produce a change in mode. It seems reasonable to conclude, therefore, that the different instructions with the familiar object also did not produce a change from a constancy to a proximal mode. Furthermore, there is other evidence that apparent instructions result in a linear or metric response to size. This evidence is found in the high correlation that occurred in the Gogel (1969) and Gogel and Newton (1969) experiments between the off-sized perceptions of the 17 familiar objects. In one experiment, the obtained values of $S_{\mathrm{c}} / S^{\prime}$ resulted from reports obtained from comparisons of size as perceived with the remembered size of a normal object of that kind. Such judgments with respect to remembered size were very likely to be metric. In the other experiment, $S_{\mathrm{c}} / S^{\prime}$ was calculated by comparing the actual familiar sizes with the size responses obtained under apparent instruction. For mean and me$\operatorname{dian} S_{\mathfrak{c}} / S^{\prime}$ values, the correlations between experiments were $r=.90$ and .98 , respectively. These results suggest that it is unlikely that apparent instructions in the present study produced a mode of response to size that did not involve a linear response and therefore did not involve the processing of information regarding distance. Instead, it is very likely that the instructions simply modified the weight given, in the response, to perceived size as opposed to cognitive (familiar) size.

Although the instructions to use apparent or objective size clearly modified the reports of size, instructions to use apparent or objective distance had little or no effect on reported distance either in the experimental situations using the playing card or blank rectangle or in the fullcue calibration situation. The latter results are consistent with those of Da Silva and Dos Santos (1984), who found that the type of instructions, using the magnitude estimation procedure, did not affect the distance responses obtained in a large open field. However, in addition to the effects of instructions on distance responses that Higashiyama (1984) found, Rogers and Gogel (1975) also found some effects under laboratory, full-cue conditions. It is not clear why distance responses were not sensitive to instructions in the present study. One possibility is that the observer can use $D_{\mathrm{c}}$ without being aware that it is a factor that should be disregarded when attempting to respond directly to apparent, as contrasted with objective, distance.

There are two different approaches to explaining spatial perceptions. One, with some legacy from the corecontext hypothesis (Boring, 1942, 1946), suggests that perception can occur in two modes. One mode, the proximal (or analytic) mode, includes perceptions that correspond directly to the core retinal stimulus without substantial modification by higher order factors such as perceived depth, perceived distance, or attention. The other, called the constancy or world mode (Rock, 1983), includes perception consistent with hypotheses of invariance, such as the SDIH. The proximal mode is postulated to occur particularly when several objects are compared in the absence of large directional separations, when the stimulus is presented for a very short time, or when distance cues are effectively reduced either by a physical reduction or by the withdrawal of attention. The constancy mode is assumed to be dominant when information as to distance is rich and is utilized by the observer. The relative weights given to these different modes is expected to be modified by instructions or by the observer's task. Modern variations of this dual-mode approach are found in Rock (1983), Epstein and Lovitts (1985), and Epstein and Broota (1986).

The other approach, advocated by the present study, denies the above distinction between proximal and constancy modes (Gogel, 1973, 1977; also see Epstein \& Broota, 1986). Instead, the change in response as a consequence of a physical or functional reduction in distance cues is attributed to the increased importance of observer tendencies, for example, the specific distance or equidistance tendency (Gogel, 1977), or to a change in weight given perceptual versus cognitive factors. The reduction in distance information, however achieved, is not expected to change the significance of perceived distance in determining other dimensions, for example, perceived size. It is expected to modify only the constellation of distance factors (e.g., the egocentric reference distance) by which the perception of distance is determined. The present study contributes to the evidence for this approach.

\section{REFERENCES}

BorING, E. G. (1942). Sensation and perception in the history of experimental psychology. New York: Appleton.

Boring, E. G. (1946). The perception of objects. American Journal of Physics, 14, 97-102.

Da Silva, J. A., Dos Santos, R. A. (1984). The effects of instructions on scales for perceived egocentric distance in a large open field. Bulletin of the Psychonomic Society, 22, 189-192.

EPSTEIN, W. (1965). Nonrelational judgments of size and distance. American Journal of Psychology, 78, 120-123.

Epstein, W., \& BrootA, K. D. (1986). Automatic and attentional components in perception of size-at-a-distance. Psychophysics, 40, 256-262.

EPSTEIN, W., \&ovitTs, B. E. (1985). Automatic and attentional components in perception of shape-at-a-slant. Journal of Experimental Psychology: Human Perception \& Performance, 3, 473-483.

EPstein, W., Park, J., \& CASEY, A. (1961). The current status of the size-distance hypothesis. Psychological Bulletin, 58, $491-514$.

FitzPatrTick, V., PASnak, R., \& TYer, Z. E. (1982). The effect of familiar size at familiar distances. Perception, 11, 85-91.

Gilinsky, A. S. (1951). Perceived size and distance in visual space. Psychological Review, 58, 460-482.

GoGEL, W. C. (1964). Size cue to visually perceived distance. Psychological Bulletin, 62, 217-235.

GOGEL, W. C. (1969). The effect of object familiarity on the perception of size and distance. Quarterly Journal of Experimental Psychology, 21, 239-247.

GoGEL, W. C. (1972). Scalar perceptions with binocular cues of distance. American Journal of Psychology, 85, 477-498.

GoGEL, W. C. (1973). The organization of perceived space II. Conse- 
quences of perceptual interactions. Psychologische Forschung, 36, 223-247.

GoGel, W. C. (1974). Cognitive factors in spatial responses. Psychologia, 17, 213-225.

GoGEL, W. C. (1976). An indirect method of measuring perceived distance from familiar size. Perception \& Psychophysics, 20, 419-429.

GoGeL, W. C. (1977). The metric of visual space. In W. Epstein (Ed.), Stability and constancy in visual perception: Mechanisms and processes. New York: Wiley.

GoGEL, W. C. (1981). The role of suggested size in distance responses. Perception \& Psychophysics, 30, 149-155.

Gogel, W. C., \& DA Silva, J. A. (1987). A two-process theory of the response to size and distance. Perception \& Psychophysics, 41, 220-238.

Gogel, W. C., \& Mertens, H. W. (1967). Perceived size and distance of familiar objects. Perceptual \& Motor Skills, 25, 213-225.

Gogel, W. C., \& NeWTon, R. E. (1969). Perception of off-sized objects. Perception \& Psychophysics, 5, 7-9.

GogeL, W. C., \& TIETZ, J. D. (1973). Absolute motion parallax and the specific distance tendency. Perception \& Psychophysics, 13, 284-292.

Granrud, C. E., HaAke, R. J., \& Yonas, A. (1985). Infants' sensitivity to familiar size: The effect of memory on spatial perception. Perception \& Psychophysics, 37, 459-466.

Hastorf, A. H. (1950). The influence of suggestion on the relationship between stimulus size and perceived distance. Journal of Psychology, 29, 195-217.

Hennessy, R. T., IidA, T., ShinNa, K., Leibowitz, H. W. (1976). The effect of pupil size on accommodation. Vision Research, 16, 587-589.

Higashiyama, A. (1984). The effects of familiar size on judgments of size and distance: An interaction of viewing attitude with spatial cues. Perception \& Psychophysics, 35, 305-312.

ItTELSON, W. H. (1951). Size as a cue to distance. American Journal of Psychology, 64, 54-67.

KILPATRICK, F. P., \& ITTELSON, W. H. (1953). The size-distance invariance hypothesis. Psychological Review, 60, 223-231.

MACK, A. (1978). Three modes of visual perception. In H. L. Peck, Jr., \& E. Saltzman (Eds.), Modes of perceiving and processing information. Hillsdale, NJ: Erlbaum.

Mershon, D. H., Kennedy, M., \& Falacara, G. (1977). On the use of calibration equations in perception research. Perception, 6, 299-311.

Mershon, D. H., LemBo, V. L. (1977). Scalar perceptions of distance in simple binocular configurations. American Journal of Psychology, 90, 17-28.
OWENS, D. A., \& LeIBOWITZ, H. W. (1976). Oculomotor adjustments in darkness and the specific distance tendency. Perception \& Psychophysics, 20, 2-9.

OWENS, D. A., \& LeIBowitz, H. W. (1983). Perceptual and motor consequences of tonic vergence. In C. M. Schor \& K. J. Ciuffreda (Eds.), Vergence eye movements: Basic and clinical aspects. Boston: Butterworths.

Park, J. N., \& Michaelson, G. J. (1974). Distance judgments under different size-information conditions. Perception \& Psychophysics, $15,57-60$.

Rix, R., Tyer, Z., \& PASNak, R. (1983). Stimulus size and visual angle in a cue-reduced experimental setting. Bulletin of the Psychonomic Society, 21, 29-30.

Rock, I. (1977). In defense of unconscious inference. In W. Epstein (Ed.), Stability and constancy in visual perception: Mechanisms and processes. New York: Wiley.

Rock, I. (1983). The logic of perception. Cambridge, MA: MIT Press. Rock, I., \& MCDERMOTT, W. (1964). The perception of visual angle. Acta Psychologica, 22, 119-134.

Rogers, S. P., \& Goges, W. C. (1975). Relation between judged and physical distance in multicue conditions as a function of instructions and tasks. Perceptual \& Motor Skills, 41, 171-178.

SchlosBerg, H. (1950). A note on depth perception, size constancy, and related topics. Psychological Review, 57, 314-317.

Yonas, A., Pettersen, L., \& Granrud, C. E. (1982). Infants' sensitivity to familiar size as information for distance. Child Development, $53,1285-1290$.

\section{NOTE}

1. It is equally appropriate to call the ratio $S_{\mathrm{c}} / S^{\prime}$ either an off-sized judgment or an off-sized perception, since it involves both a memory $\left(S_{\mathrm{c}}\right)$ and a perceptual $\left(S^{\prime}\right)$ component (see Gogel, 1981, Footnote 1). If it is termed an off-sized perception, this should not be taken as meaning that familiar size $\left(S_{\mathrm{c}}\right)$ has modified a perception. The ratio $S_{\mathrm{c}} / S^{\prime}$ can be changed by changing $S_{\mathrm{c}}$ without implying a change in the perceived size $\left(S^{\prime}\right)$. Thus, the effect of $S_{\mathrm{c}}$ upon the ratio $S_{\mathrm{c}} / S^{\prime}$ does not mean, as it seems to be interpreted by Granrud, Haake, and Yonas (1983, p. 463), that the theory expressed here "assumes that familiar size can influence perceived size without influencing perceived distance."

(Manuscript received November 7, 1983; revision accepted for publication December 18, 1986). 